\title{
Análise da produção de lágrima em pacientes com alopecia periocular: estudo retrospectivo entre os anos 2008 e 2012 no Hospital Veterinário da Universidade Estadual de Londrina
}

\section{Analysis of tear production in patients with periocular alopecia: a retrospective study between the years 2008 and 2012 at the Veterinary Hospital of the Londrina State University}

\author{
Maria Fernanda de Souza Ferraz ${ }^{1 *}$; Mirian Siliane Batista de Souza ${ }^{2}$
}

\begin{abstract}
Resumo
A Ceratoconjuntivite Seca é uma oftalmopatia frequente entre os cães e pode estar acompanhada de alopecia periocular, decorrente da extensão ou concomitância da inflamação palpebral. No estudo foram analisados prontuários de cães com Ceratoconjuntivite Seca e Blefarites atendidos no Hospital Veterinário da Universidade Estadual de Londrina entre os anos de 2008 e 2012, para buscar pacientes com alopecia periocular, verificando a frequência destas enfermidades na população em relação ao gênero, idade, raça, olho acometido, outras afecções oftálmicas concomitantes, possíveis etiologias e presença de doenças sistêmicas nestes cães. A partir da análise dos dados dos animais com as oftalmopatias referidas, pretendeu-se discutir a relação entre a ocorrência de alopecia periocular e diminuição da produção lacrimal, além de possíveis causas, alterações oculares presentes, enfermidades sistêmicas, raças acometidas, gênero e idade. O Teste Lacrimal de Schirmer foi o método utilizado para aferição da produção lacrimal. Foram encontrados 187 cães, dos quais 147 apresentaram ceratoconjuntivite seca e 71 blefarite. Em 31 cães as enfermidades apresentaram-se associadas. Ocorreram 41 casos de alopecia periocular sem diferença na frequência de acometimento em relação ao gênero dos pacientes. Quanto ao olho acometido, $95 \%$ dos casos mostram-se bilateral. As raças que tiveram maior prevalência foram Poodle, Lhasa Apso, Cocker Spaniel Inglês e cães sem raça definida. As idades mais frequentes foram dois anos, com 19\% dos animais, quatro e 12 anos com 12\%. Dos 32 animais com alopecia periocular que apresentaram doenças sistêmicas, 22 tinham dermatopatias. Concluiu-se que a ceratoconjuntivite seca e a blefarite não aparecem associadas com tanta frequência e que a ocorrência de alopecia periocular se dá na maior parte, em casos de blefarite, embora possa, com menor frequência, apresentar-se na CCS. Apesar da produção de lágrima exibir-se diminuída em alguns pacientes com alopecia periocular, não se pode dizer que sempre esteja presente nestes casos.
\end{abstract}

Palavras-chave: Cães, blefarite, ceratoconjuntivite seca, filme lacrimal

\begin{abstract}
The Keratoconjunctivitis Sicca is a common ophthalmopathy among dogs and can be accompanied by periocular alopecia resulting from the extension of inflammation and concomitant eyelid. In the study, medical records of dogs with keratoconjunctivitis sicca and blepharitis treated at the Veterinary Teaching Hospital of the Londrina State University between the years 2008 and 2012 were analyzed, seeking patients with periocular alopecia and checking the frequency of these diseases in the population
\end{abstract}

\footnotetext{
${ }^{1}$ Discente de Graduação em Medicina Veterinária, Universidade Estadual de Londrina, UEL, PR. E-mail: fenderfer@gmail.com

${ }^{2}$ Médica Veterinária, Prof ${ }^{a}$ Dr $^{\mathrm{a}}$ Associada do Dept ${ }^{\mathrm{o}}$ de Clínicas Veterinárias, UEL, Londrina, PR. E-mail: msiliane@uel.br

* Autor para correspondência
} 
in relation to gender, age, breed, affected eye, other concomitant ophthalmic disorders, possible etiologies and the presence of systemic diseases. From the analysis of data it was intended to discuss the relationship between the occurrence of periocular alopecia and decreased tear production and possible causes, ocular present, systemic illnesses, affected breeds, gender and age. The Schirmer Tear Test was the method used to measure the tear production. 187 dogs were studied, 147 of which had keratoconjunctivitis sicca and 71 had blepharitis. 31 dogs showed associated diseases. There were 41 cases of alopecia periocular with no difference in the frequency of involvement in relation to gender. In regard to the affected eye, $95 \%$ of cases were bilateral. The breeds with higher prevalence were Poodle, Lhasa Apso, English Cocker Spaniel and mixed breed dogs. The ages with higher occurrence were two years, with $19 \%$ of the animals, four and twelve years with $12 \%$. Among the 32 animals with periocular alopecia who had systemic diseases, 22 had dermatopathies. It was concluded that keratoconjunctivitis sicca and blepharitis are not frequently associated and the occurrence of periocular alopecia occurs in most cases of blepharitis, although it may less frequently present itself in the CCS. Despite the decreased tear production displayed in some patients with periocular alopecia, one cannot say that it is always present in these cases.

Key words: Dogs, blepharitis, keratoconjunctivitis sicca, tear film

\section{Introdução}

A Ceratoconjuntivite Seca (CCS) ou "olho seco" é uma doença comum e o cão é a espécie mais acometida. Embora não seja uma enfermidade primária da córnea é nela que as manifestações ocorrem, como consequencia da baixa ou ausência da produção de lágrima. A afecção se caracteriza por inflamação crônica das glândulas lacrimais, córnea e conjuntiva, decorrente de alterações qualitativas e/ou quantitativas do filme lacrimal pré-corneal (FLP). Com isso, define-se esta enfermidade como diminuição da produção de lágrima. Geralmente, há alterações do componente aquoso do filme lacrimal, mas também pode decorrer de deficiência dos componentes lípidico e mucoso (STADES et al., 1999a; GELATT, 2003b; HERRERA, 2008b; WOUK; SOUZA; FARIAS, 2009).

Em associação à CCS pode-se ter a manifestação de blefarite, a qual pode estar acompanhada de alopecia periocular (STADES et al., 1999a; GELATT, 2003a; HERRERA, 2008a; GALERA; LAUS; ORIÁ, 2009). Blefarite se trata de inflamação das pálpebras e a maioria dos casos está associada a dermatopatias de diferentes origens, entre elas: alérgicas, síndrome seborréica, autoimunes, infecciosas e parasitárias. Grande parte dos cães tem alterações no filme lacrimal como consequência da reação da inflamação cutânea nas glândulas lacrimais (PEÑA; ROURA; DAVIDSON, 2000; GELATT, 2003b; PEÑA; LEIVA, 2008; HERRERA, 2008b; WOUK; SOUZA; FARIAS, 2009). A alopecia periocular pode estar presente em blefarites de qualquer origem e geralmente se apresenta na fase crônica da enfermidade (HERRERA, 2008a).

A finalidade desse trabalho foi realizar um estudo retrospectivo com análise dos prontuários dos cães atendidos no Hospital Veterinário da Universidade Estadual de Londrina (HV-UEL) com CCS associado ou não a blefarite, observando a produção de lágrima, alterações oculares e perioculares, ocorrência de doenças sistêmicas associadas, frequência das afecções de acordo com a raça, gênero e idade; e possíveis etiologias das afecções. O objetivo específico foi encontrar a relação entre a diminuição da produção de lágrima e a manifestação de alopecia periocular.

\section{Material e Métodos}

Utilizou-se um estudo retrospectivo de prontuários de cães com afecções oculares atendidos no Hospital Veterinário da Universidade Estadual de Londrina entre os anos de 2008 e 2012. Foram encontrados 187 animais, sem preferência por raça, gênero ou idade, analisou-se os prontuários de 
pacientes com CCS ou blefarites independentemente dos fatores citados.

O Teste da Lágrima de Schirmer (TLS) foi o método padrão empregado para aferição da produção de lágrima utilizado no exame oftalmológico destes animais. O teste considerado neste estudo foi sempre o TLS 1 (sem o uso de colírio anestésico).
Para que houvesse padronização dos dados analisados nas fichas, as informações sobre os pacientes foram colocadas em um Quadro de Colheita de Dados (Tabela 1) previamente elaborado. Utilizou-se uma tabela individual, a qual foi posteriormente analisada para apresentação dos resultados e discussão.

Tabela 1. Colheita de Dados.

\begin{tabular}{|c|c|c|c|c|}
\hline \multicolumn{5}{|l|}{ Dados do Animal } \\
\hline RG: & Nome: & Idade: & Sexo: & Raça: \\
\hline Globo Ocular acometido & GOD: & GOE: & Ambos: & \\
\hline \multicolumn{5}{|l|}{ Diagnóstico: } \\
\hline Produção lacrimal (TLS1) & GOD: & GOE: & & \\
\hline \multicolumn{5}{|l|}{ Alterações oculares: } \\
\hline \multicolumn{5}{|l|}{ Alterações perioculares: } \\
\hline Alopecia Periocular & Presente: & Ausente: & & \\
\hline Secreção & Presente: & Ausente: & & \\
\hline \multicolumn{5}{|l|}{ Tipo de Secreção: } \\
\hline \multicolumn{5}{|l|}{ Evolução do quadro: } \\
\hline \multicolumn{5}{|l|}{ Possível Etiologia: } \\
\hline \multicolumn{5}{|l|}{ Tratamento Prescrito: } \\
\hline Doença Sistêmica Presente: & & & & \\
\hline
\end{tabular}

Fonte: Elaboração dos autores.

\section{Resultados e Discussão}

Encontrou-se 187 pacientes entre 2008 e 2012 com ceratoconjuntivite seca (CCS) e blefarite, destes, 147 casos com CCS e 71 com blefarite. Em 31 animais as enfermidades apresentaram-se associadas. A manifestação de alopecia periocular foi verificada em 41 animais, 51\% fêmeas (21 casos) e $49 \%$ machos (20 casos).

A ocorrência das afecções em relação ao gênero foi de 110 fêmeas e 77 machos; sendo 92 fêmeas $(62 \%)$ e 54 machos (38\%) apresentando CCS, 37 fêmeas (52\%) e 34 machos (48\%) apresentando blefarite. Segundo Stades et al.a (1999a), a CCS ocorre mais frequentemente em fêmeas (cerca de 65\%). Em particular, andrógenos são potentes estimuladores da produção lacrimal com importantes efeitos relacionados ao gênero na produção de lágima (STADES et al., 1999a; WILLIAMS, 2008). Observa-se assim, proximidade dos dados encontrados no estudo com os descritos na literatura. Na blefarite não foram encontrados dados para análise comparativa.

Quanto ao olho acometido por CCS, a partir da análise dos prontuários, foram encontrados $84 \%$ dos casos (123) manifestando-se bilateral, enquanto unilateral em 9\% (13) apresentaram-se no olho direito e, 7\% (11) no olho esquerdo. De acordo Stades et al. (1999a), a ceratoconjuntivite seca geralmente é bilateral, manifestando-se em $60 \%$ dos animais. Aproximam-se, assim, os dados colhidos com os descritos pelo referido autor.

Entre os casos de blefarite, foram observados 
86\% (61) com apresentação bilateral, 7\% (cinco) manifestando-se apenas no olho direito e $7 \%$ (cinco) no olho esquerdo. Segundo Peña e Leiva (2008), geralmente é bilateral. Dessa forma, equiparam-se os resultados obtidos com a literatura.

Dos 147 cães com CCS as raças encontradas foram: Poodle (20), Lhasa Apso (19), Shitzu (15), Cocker Spaniel Inglês (13), Cocker Spaniel Americano (12), Pinscher (12), Maltês (quatro), Yorkshire Terrier (quatro), Akita (dois), Labrador (dois), Rottweiler (dois), Pastor Alemão (dois), Pitbull (dois), Schnauzer (dois), Basset Hound (um), Beagle (um), Boxer (um), Dachshund (um), Pequinês (um), Sharpei (um) e West Highland White Terrier (um) e 29 cães sem raça definida (SRD). Com relação à CCS, Giuliano e Moore (2007) relatam que qualquer raça pode apresentar a enfermidade, mas as acometidas com maior frequência são: Bloodhound, Boston Terrier, Buldogue Inglês, Cavalier King Charles Spaniel, Cocker Spaniel Americano, Lhasa Apso, Samoyeda, Schnauzer miniatura, Shitzu, Pequinês, Pug e West Highland White Terrier. De acordo com Stades et al. (1999a), a predisposição racial inclui as de pequeno porte em geral e também o Dachshund pêlo longo. Em concordância com a literatura, as raças com predisposição genética encontradas no estudo são: Cocker Spaniel Americano, Dachshund, Lhasa Apso, Schnauzer, Shitzu, Pequinês, West Highland White Terrier e os cães de raças pequenas: Poodle, Pinscher, Maltês, Yorkshire Terrier e Pequinês. No Brasil os SRD são muito populares e talvez, por esse motivo, se explique a sua alta incidência.

Dos 71 cães com blefarite, a frequência de raças encontradas foi: Poodle (nove), Lhasa Apso (sete), Shitzu (sete), Cocker Spaniel Americano (cinco), Pitbull (quatro), Maltês (três), Cocker Spaniel Inglês (dois), Dachshund (dois), Labrador (dois), Schnauzer (dois), Yorkshire Terrier (dois), Basset Hound (um), Beagle (um), Boxer (um), Bull Terrier (um), Fila Brasileiro (um), Pinscher (um), Rotweiller (um), Sharpei (um) e 18 cães SRD. Peña e Leiva (2008) descrevem a enfermidade blefarite como principais raças predispostas: Pastor Alemão, Poodle, Dachshund de pêlos longos (blefarites autoimunes); Labrador, Lhasa Apso, Dachshund, Golden Retriever, Gordon Retriever (blefarite secundária a celulite juvenil); West Highland White Terrier (blefarite alérgica). Portanto, em concordância com a literatura as raças encontradas na pesquisa são: Dachshund, Lhasa Apso e Poodle.

$\mathrm{Na}$ CCS, foram encontrados cães com idade entre três meses e 18 anos, observando maior prevalência aos sete anos (13). Entre os casos a ocorrência encontrada foi: menos de um ano (seis), um ano (nove), dois anos (oito), três anos (12), quatro anos (12), cinco anos (sete), seis anos (11), oito anos (10), nove anos (10), dez anos (oito), 11 anos (nove), 12 anos (oito), 13 anos (oito), 14 anos (dois), 15 anos (dois), 16 anos (um), 17 (dois) e 18 anos (um).

$\mathrm{Na}$ blefarite a maior incidência aconteceu aos dois anos com 11 casos (15\%) e a idade variou de um a 12 anos. Foram encontrados: um ano (três), três anos (três), quatro anos (10), cinco anos (dois), seis anos (dois), sete anos (cinco), oito anos (cinco), nove anos (dois), dez anos (seis), 11 anos (quatro), 12 anos (sete).

Nos casos em que a CCS e a blefarite se apresentaram associadas, a variação de idade foi de três meses a 14 anos. Com menos de um ano (três), um ano (um), dois anos (três), três anos (nenhum), quatro anos (três), cinco anos (nenhum), seis anos (um), sete anos (dois), oito anos (três), nove anos (um), 10 anos (dois), 11 anos (três), 12 anos (três), 13 anos (dois), 14 anos (um).

Tanto a CCS quanto a blefarite podem acometer animais de qualquer idade, uma vez que apresentam diversas etiologias (STADES et al., 1999a).

As manifestações oculares encontradas nos pacientes estudados foram: opacidade de córnea (98), congestão de vasos episclerais (93), neovascularização em córnea (75), hiperemia conjuntival (74), hiperpigmentação em córnea (63), quemose (42), opacidade de lente (38), diminuição 
da visão (18), leucoma cicatricial (11), hifema (oito), miose puntiforme (oito), midríase não responsiva (sete), diminuição de reflexo pupilar (sete), cegueira (quatro), retinopatias (quatro), anisocoria (quatro), hipópio (três), sinéquia anterior (três), estafiloma (dois), prolapso de íris (dois), hiperpigmentação de lente (um), heterocromia de íris (um) e nistagmo (um). Wouk, Souza e Farias (2009) descrevem que além da diminuição da produção de lágrima no TLS 1, a CCS manifesta sinais clínicos como: perda do brilho da superfície corneal, hiperemia conjuntival, secreção ocular mucosa, desconforto, vascularização, pigmentação, blefaroespasmo e perda da visão; correspondendo ao encontrado nos resultados deste trabalho.

Dos 41 animais que apresentaram alopecia periocular, 33 mostraram blefarite e 19 CCS. As demais alterações perioculares encontradas foram: 31 com blefaroespasmo, $21 \mathrm{com}$ edema de pálpebras, 16 com prurido periocular, 13 com lesões ou crostas perioculares, 12 com hiperemia palpebral, nove com epífora, nove com fotofobia, três com hiperqueratose e hiperpigmentação palpebral. Herrera (2008a) descreve que muitos cães acabam sendo levados à consulta com quadros de epífora ou com leves quantidades de secreções amareladas devido a presença de blefarites. De acordo com Gelatt (2003b) e Stades et al.(1999b) devido às pálpebras serem altamente vascularizadas, a hiperemia e o edema são intensos, acompanhados ou não de prurido. A dor é indicada pelo blefaroespasmo e lacrimejamento excessivo, podendo também se observar a presença de exsudato, lesões por auto traumatismo e descamação. Em casos de inflamação palbebral crônica, pode ocorrer deformação, com entrópio ou ectrópio. Conforme os resultados encontrados, assim como na literatura, temos uma confirmação de relação entre os dados.

A presença de secreção foi constatada em $91 \%$ dos casos (171). Desses, 102 casos com tipo purulenta, sendo $66 \%$ em casos de CCS e $34 \%$ em de blefarite; em 27 com tipo mucopurulenta, em que 70\% ocorreram nos casos de CCS e 30\% nas blefarites;
16 com tipo mucóide, com $75 \%$ aparecendo no CCS e $25 \%$ na blefarite, e 18 com tipo serosa, com $56 \%$ em CCS e $44 \%$ em blefarite. Em oito casos o tipo de secreção não foi especificado. Segundo Gelatt (2003b) na maioria dos casos de CCS, há presença de secreção mucóide ou mucopurulenta intermitente, conforme o quadro se agrava, a descarga ocular se torna mucopurulenta persistente. Neste estudo, as secreções mucopurulenta e mucoide apresentam-se com elevada incidência (70\% e $75 \%$, respectivamente), em acordo com o citado autor. Em relação à blefarite não há dados para a comparação.

Em relação a alopecia periocular, sua manifestação foi bilateral em 95\% dos casos (39), desses, dois casos (uma fêmea e um macho) com apresentação unilateral no olho direito. Quanto à raça, tivemos 13 SRD (seis fêmeas e sete machos); seis Poodle (duas fêmeas e quatro machos); cinco Lhasa Apso (duas fêmeas e três machos); três Cocker Spaniel Inglês (três fêmeas); dois Shitzu (uma fềmea e um macho); dois Pitbull (uma fêmea e um macho); dois Labrador (uma fêmea e um macho); um Cocker Spaniel Americano (fêmea); um Dachshund (fềmea); um Sharpei (fêmea); um Bull Terrier (fêmea); um Boxer (macho); um Beagle (macho); e um Pinscher (macho). A idade dos animais com alopecia periocular variou de três meses a 13 anos. Dois anos foi a idade de maior prevalência (8 casos). As idades de 4 e 12 anos apareceram em cinco casos; 6, 7 e 11 anos com três casos; 1 e 10 anos com dois casos; e as demais com um caso cada uma. Em 35 casos de alopecia periocular foi relatada presença de secreção nos olhos: 24 com tipo purulenta, seis com tipo serosa, quatro com tipo mucopurulenta, três com tipo mucóide, três sem secreção e um com o tipo de secreção não especificado.

Nos 31 casos em que foram encontradas CCS e blefarite associadas, 19 eram fêmeas $(61 \%)$ e 11 eram machos (39\%). A raça de maior prevalência foi o Lhasa Apso (seis), seguida por Poodle (cinco), Cocker Spaniel Americano (quatro), Shitzu (três), 
Maltês (dois), Pitbull (dois), Basset Hound (um), Cocker Spaniel Inglês (um), Schnauzer (um), Sharpei (um) e cinco cães SRD.

O Teste Lacrimal de Schirmer (TLS) não foi realizado em 49 pacientes (26 com CCS e $23 \mathrm{com}$ blefarite). Nestes pacientes, o diagnóstico de CCS foi baseado no achado clínico de superfície ocular evidentemente seca. Dos cães com blefarite, 18 tiveram o TLS1 realizado. Foi observado no olho direito: 10 abaixo de $5 \mathrm{~mm} / \mathrm{min}$; nove entre 5 e 9 $\mathrm{mm} / \mathrm{min}$; nove entre 10 e $14 \mathrm{~mm} / \mathrm{min}$ e 19 acima de $15 \mathrm{~mm} / \mathrm{min}$. No olho esquerdo foi observado: 12 abaixo de $5 \mathrm{~mm} / \mathrm{min} ; 11$ entre 5 e $9 \mathrm{~mm} / \mathrm{min} ; 12$ entre 10 e $14 \mathrm{~mm} / \mathrm{min}$ e 12 acima de $15 \mathrm{~mm} / \mathrm{min}$.

Em 121 casos de CCS foi realizado o TLS1, observando-se no olho direito: 49 deles com produção lacrimal abaixo de $5 \mathrm{~mm} / \mathrm{min} ; 35 \mathrm{com}$ produção lacrimal entre 5 e $9 \mathrm{~mm} / \mathrm{min} ; 20$ com produção lacrimal entre 10 e $14 \mathrm{~mm} / \mathrm{min}$ e, 16 com produção lacrimal acima de $15 \mathrm{~mm} / \mathrm{min}$. No olho esquerdo verificou-se: 46 deles com produção lacrimal abaixo de $5 \mathrm{~mm} / \mathrm{min} ; 28 \mathrm{com}$ produção lacrimal entre 5 e $9 \mathrm{~mm} / \mathrm{min}$; 26 com produção lacrimal entre 10 e $14 \mathrm{~mm} / \mathrm{min}$ e, 20 com produção lacrimal acima de $15 \mathrm{~mm} / \mathrm{min}$.

Nos episódios em que as enfermidades mostraram-se associadas (CCS e blefarite), a produção lacrimal observada a partir da realização do TLS1, apresentou, nos olhos direitos: 10 abaixo de $5 \mathrm{~mm} / \mathrm{min}$; sete entre 5 e $9 \mathrm{~mm} / \mathrm{min}$; cinco entre 10 e $14 \mathrm{~mm} / \mathrm{min}$ e, sete acima de $15 \mathrm{~mm} / \mathrm{min}$. Nos olhos esquerdos: 10 abaixo de $5 \mathrm{~mm} / \mathrm{min}$; oito entre 5 e $9 \mathrm{~mm} / \mathrm{min}$; nove entre 10 e $14 \mathrm{~mm} / \mathrm{min}$ e, dois acima de $15 \mathrm{~mm} / \mathrm{min}$.

Em relação aos casos de alopecia periocular, 13 não tiveram o TLS1 realizado; nos 28 restantes foi constatada a produção lacrimal nos olhos direitos: nove abaixo de $5 \mathrm{~mm} / \mathrm{min}$; quatro entre 5 e $9 \mathrm{~mm} /$ $\mathrm{min}$; oito entre 10 e $14 \mathrm{~mm} / \mathrm{min}$ e sete acima de 15 $\mathrm{mm} / \mathrm{min}$. Nos olhos esquerdos: sete abaixo de $5 \mathrm{~mm} /$ $\mathrm{min}$; seis entre 5 e $9 \mathrm{~mm} / \mathrm{min} ; 10$ entre 10 e $14 \mathrm{~mm} /$ min e, cinco acima de $15 \mathrm{~mm} / \mathrm{min}$.
Nos animais (oito) que não apresentaram alterações oculares descritas nos prontuários, apenas três tiveram o TLS realizado, sendo que dois tiveram a produção bilateral acima de $20 \mathrm{~mm} / \mathrm{min}$ e um apresentou produção lacrimal entre 5 e $9 \mathrm{~mm} /$ min no olho direito e, abaixo de $5 \mathrm{~mm} / \mathrm{min}$ no olho esquerdo.

De acordo com a literatura, valores normais descritos para o TLS 1 situam-se entre 18,9 e 23,9 $\mathrm{mm}$ para o cão. Valores menores que $5 \mathrm{~mm} / \mathrm{min}$ são considerados CCS e valores menores que $10 \mathrm{~mm} /$ min são considerados suspeitos, mas se combinados com sinais clínicos de ceratoconjuntivite seca são considerados como confirmatórios da afecção (GELATT, 2003b). Segundo Herrera (2008a) a maioria dos pacientes com blefarites apresentam alterações evidentes no filme lacrimal devido às mudanças consequentes da inflamação palpebral.

Concomitantemente, em ocorrência de CCS associada ou não à blefarite, foram observadas outras oftalmopatias nos pacientes analisados, dentre elas: ceratite ulcerativa (59), entrópio (22), catarata (19), distiquíase (19), neoformação palpebral (10), protrusão de glândula 3. a pálpebra (oito), esclerose de lente (sete), uveíte (sete), cílio ectópico (seis), ceratite pigmentar (cinco), miíase em globo ocular (cinco), phithisis bulbi (cinco), conjuntivite (cinco), glaucoma (quatro), triquíase (quatro), atrofia senil de íris (dois), calázio (dois), ceratite (três), ectrópio (dois), estafiloma (dois), úlcera corneana em melting (dois), ceratite bolhosa (um), distrofia de córnea (um), endoftalmite (um), Florida spots (um), luxação de lente (um), protrusão de globo ocular (um), SARD - Degeneração súbita adquirida da retina (um), úlcera indolente do Boxer (um). Segundo Gelatt (2003b) os sinais clínicos de CCS variam dependendo do tempo decorrido do surgimento e extensão do ressecamento da córnea. Numa forma aguda e severa o olho se torna doloroso em associação à ceratite ulcerativa (STADES et al., 1999a; GELATT, 2003b; HERRERA, 2008b); GALERA; LAUS; ORIÁ, 2009). Em comparação à literatura foi encontrada 
uma prevalência significativa de úlcera corneal; já com relação às blefarites, encontrou-se presença de entrópio e ectrópio. De acordo com Gelatt (2003b) nos casos de doença corneal progressiva, verifica-se descemetocele com resultante estafiloma e prolapso de íris; os dois primeiros também foram encontrados nos pacientes estudados.

Levando-se em conta que alguns cães apresentaram mais de uma oftalmopatia simultaneamente, entre os presentes nos casos de CCS associados à blefarite, destacaram-se: ceratite ulcerativa (14), catarata (quatro), entrópio (três), distiquíase (três), cílio ectópico (dois) e nos demais, cada um com as seguintes enfermidades: protrusão de glândula da terceira pálpebra, triquíase, esclerose de lente, calázio, hifema e atrofia senil de íris. Com base na literatura consultada não foram encontrados elementos passíveis de comparação.

Dos animais com alopecia periocular que apresentaram simultaneamente CCS e/ou blefarite entre as oftalmopatias encontradas destacam-se: ceratite ulcerativa (oito), catarata (quatro), entrópio (três), distiquíase (três), esclerose de lente (três), conjuntivite (dois), dois com uveíte (dois), ceratite pigmentar (dois), neoformação palpebral (um), calázio (um), distrofia de córnea (um), protrusão de terceira pálpebra (um), triquíase (um), hifema (um) e atrofia senil de íris (um). Apenas em oito animais com alopecia periocular não foram descritas outras alterações oculares dessa doença. Na literatura consultada não foram encontrados dados que possibilitem comparação.

Quanto à presença de doenças sistêmicas que podem ser consideradas como possíveis etiologias, destacam-se a cinomose, dermatopatias e doenças endócrinas. $\mathrm{Na}$ análise dos prontuários foram encontrados 39 casos suspeitos de cinomose, sendo que destes, apenas dois foram confimados por PCR (reação em cadeia da polimerase). Em relação às dermatopatias foi verificado um total de 58 casos, e em oito pacientes observou-se seborreia seca, sete com atopia, sete com piodermatite bacteriana, cinco com sarna demodécica, cinco com dermatite alérgica à saliva de pulga (DASP), cinco com dermatite úmida aguda, dois com dermatite interdigital, um com dermatite fúngica, um com seborreia oleosa, um com dermatite em prega nasal, um com dermatite escrotal e um com dermatite perivulvar. Os 14 demais casos não tiveram o diagnóstico claramente especificado.

Entre as alterações dermatológicas descritas, foram encontradas: alopecia generalizada (11), rarefação pilosa (10), lesões e crostas, descamação, colaretes epidérmicos, pústulas, eritema, hiperqueratose, hipotricose e prurido. A ocorrência de ectoparasitas (pulgas e carrapatos), nestes pacientes, foi de seis e quatro casos, respectivamente. Entre as doenças endócrinas, observou-se um caso confirmado e um caso suspeito de diabete melito, sendo que, apenas o caso suspeito apresentou alterações dermatológicas; por outro lado, ambos apresentaram CCS. Foram também observados dois casos de hiperadrenocorticismo, mas sem alterações dermatológicas descritas no prontuário do paciente. A otite foi uma enfermidade que apareceu com prevalência significativa, com um total de 35 casos.

Em comparação aos resultados encontrados na pesquisa, ressalta-se, conforme descrito por Herrera (2008a), que mais de 90\% dos Cockers spaniels com olho seco apresentam seborreia, e mais de $70 \%$ de Shitzus e Lhasa apsos com CCS sofrem de atopia. Estas dermatopatias afetam as pálpebras e produzem mudanças na composição do filme lacrimal pré-corneal.

Nos cães que apresentam CCS e blefarite associadas, 17 deles exibiam dermatopatias (13 fêmeas e quatro machos) e cinco foram suspeitos de cinomose (duas fêmeas e três machos). Segundo Gelatt (2003b), em associação à CCS, pode-se ter a manifestação de blefarite e dermatite periocular, com acúmulo de exsudatos nas margens palpebrais e na pele periocular, confirmando a presença de dermatopatias nos casos de blefarite, as quais, muitas vezes, são responsáveis por alterar o filme 
lacrimal, desencadeando quadros de CCS.

Nos animais com alopecia periocular, 32 apresentaram doença sistêmica associada ao quadro oftálmico de CCS e/ou blefarite, sendo 22 destes dermatopatas (15 fêmeas e sete machos), cinco suspeitos de cinomose (quatro fêmeas e um macho) e os demais com outras enfermidades não significativas.

Apenas 34 animais não têm nenhuma doença sistêmica registrada em seus prontuários, sendo atendidos no HV-UEL apenas devido às suas alterações oculares.

Embora sem importância clínica em relação às oftalmopatias em estudo, destaca-se a ocorrência de algumas doenças sistêmicas nos pacientes pesquisados, entre elas: cardiopatias (sopro de mitral, arritmia sinusal), afecções neurológicas (convulsão, encefalite viral, déficit de nervos cranianos, síndrome cerebelar, síndrome vestibulo-central, síndrome multifocal), afecções ortopédicas (atropelamentos, traumatismo crânio-encefálico, luxação de patela, displasia coxofemoral, fraturas, osteoartrites, ruptura de ligamento cruzado), afecções no sistema reprodutor (piometra, hemometra, distocia, tumor venéreo transmissível, tumor de mama, mastite, pseudociese, criptorquidismo, prostatomegalia), afecções no sistema urinário (insuficiência renal crônica, cístite), afecções orais (doença periodontal, papiloma oral, fratura dentária, úlcera em língua, hiperplasia gengival, prognatismo), afecções no sistema digestório (verminoses, gastroenterite hemorrágica, corpo estranho intestinal, pancreatite), hepatopatias (insuficiência hepática, hepatomegalia, neoplasia), afecções no sistema respiratório (pneumonia, bronquite crônica, metástase pulmonar, colapso de traquéia), neoplasias, miíase, abscessos, feridas por mordedura, efusão (pleural, abdominal), inflamação de glândula perianal, septicemia, intoxicações (piretróides, varfarina), hérnias (inguinal, umbilical), mucocele (cervical, submandibular), toxoplasmose e erliquiose.
Conforme descrito por Gelatt (2003a) animais enfermos podem ter sua produção de secreção de componentes da lágrima diminuídos por terem os tecidos secretores direta ou indiretamente afetados, por exemplo: lesões traumáticas nas glândulas lacrimais ou na sua inervação (base da orelha), não correção da glândula terceira pálpebra protruída ou remoção cirúrgica desta, doenças metabólicas sistêmicas (hipotiroidismo, diabete melito e doença de Cushing), vírus da cinomose, tratamentos prolongados com medicações a base de sulfa ou colírio de atropina, blefaroconjuntivite crônica, doenças imunomediadas, congênitas e predisposição genética. Entre outras causas, foram descritos por Stades et al. (1999a), a deficiência nutricional de vitamina A, botulismo, doenças degenerativas e causa idiopática.

Contudo, a partir dos resultados encontrados, observou-se que a ceratoconjuntivite seca e a blefarite não apareceram associadas com frequência expressiva. Na CCS, a prevalência em fêmeas foi claramente verificada, enquanto a blefarite não apresentou diferença de acometimento em relação ao gênero. A alopecia periocular ocorreu na maior parte em casos de blefarite, embora possa, com menor frequência, apresentar-se na CCS.

\section{Conclusões}

Apesar da produção de lágrima mostrar-se diminuída em alguns pacientes com alopecia periocular, não se pode afirmar que sempre esteja presente nestes casos. Conclui-se, portanto, dentro da metodologia aplicada, que não houve relação direta entre a diminuição da produção de lágrima e alopecia periocular.

\section{Referências}

GALERA, P. D.; LAUS, J. L.; ORIÁ, A. P. Afecções da túnica fibrosa. In: LAUS, J. L. Oftalmologia clínica e cirúrgica em cães e gatos. São Paulo: Editora ROCA, 2009. p. 69-96. 
GELATT, K. N. Doenças e cirurgia das pálpebras do cão. In:_. Manual de oftalmologia veterinária. Barueri: Editora Manole Ltda, 2003a. p. 47-72.

Doenças e cirurgia dos sistemas lacrimal e nasolacrimal do cão. In:__. Manual de oftalmologia veterinária. Barueri: Editora Manole Ltda, 2003b. p. 7394.

GIULIANO, E. A.; MOORE, C. P. Diseases and surgery of the lacrimal secretory system. In: . Veterinary ophthalmology. 4. ed. Iowa: Blackwell Publishing, 2007. p. 633-661.

HERRERA, D. H. Enfermidades palpebrais. In: Oftalmologia clínica em animais de companhia. São Paulo: Editora MedVet, 2008a. p. 89-100.

. Afecções da córnea. In: . Oftalmologia clínica em animais de companhia. São Paulo: Editora MedVet, 2008b. p. 111-140.

PEÑA, M. T.; LEIVA, M. Canine conjunctivitis and blepharitis. In: WILLIAMS, D. L. Veterinary clinics: small animal practice. Barcelona: Elsevier Saunders, 2008 , v. 38 , p. 233-249.
PEÑA, M. T.; ROURA, X.; DAVIDSON, M. G. Ocular and periocular manifestations of leishmaniosis in dogs: 105 cases (1993-1998). Veterinary Ophthalmology, 2000, Barcelona, v. 3, p. 35-41.

STADES, F. C.; BOEVÉ, M. H.; NEUMANN, W.; WYMAN, M. Aparelho Lacrimal. In: STADES, F. C.; BOEVÉ, M. H.; NEUMANN, W.; WYMAN, M. Fundamentos de oftalmologia veterinária. São Paulo: Editora Manole Ltda, 1999a. p. 54-63.

. Pálpebras. In: STADES, F. C.; BOEVÉ, M. H.; NEUMANN, W.; WYMAN, M. Fundamentos de oftalmologia veterinária. São Paulo: Editora Manole Ltda, 1999b. p. 64-88.

WILLIAMS, D. L. Imunopathogenesis of keratoconjunctivitis sicca in the dog. In: Veterinary clinics: small animal practice. England: Elsevier Saunders, 2008, v. 38, p. 251-268.

WOUK, A. F. P. F.; SOUZA, A. L. G.; FARIAS, M. R. afecções dos anexos oftálmicos. In: LAUS, J. L. Oftalmologia clínica e cirúrgica em cães e gatos. São Paulo: Editora ROCA, 2009. p. 33-68. 
